OPEN ACCESS

Edited by: Daisuke Yabe,

Gifu University, Japan

Reviewed by:

Zhimin Huang,

The First Affiliated Hospital of

Sun Yat-sen University, China

Yimin Zhu,

Zhejiang University, China

*Correspondence:

Jinghong Zhao

zhaojh@tmmu.edu.cn

Jiachuan Xiong

xiongjc@tmmu.edu.cn

${ }^{\dagger}$ These authors have contributed equally to this work and share

the first authorship

Specialty section: This article was submitted to Clinical Diabetes,

a section of the journal

Frontiers in Endocrinology

Received: 26 June 2021 Accepted: 15 December 2021

Published: 14 January 2022

Citation:

Li H, Shen Y, Yu Z, Huang Y, He T, Xiao T, Li Y, Xiong J and Zhao J (2022)

Potential Role of the Renal Arterial

Resistance Index in the Differential Diagnosis of Diabetic Kidney Disease.

Front. Endocrinol. 12:731187. doi: 10.3389/fendo.2021.731187

\section{Potential Role of the Renal Arterial Resistance Index in the Differential Diagnosis of Diabetic Kidney Disease}

\author{
Haiyang $\mathrm{Li}^{\dagger}$, Yunzhu Shen ${ }^{\dagger}$, Zhikai Yu, Yinghui Huang, Ting He, Tangli Xiao, Yan Li, \\ Jiachuan Xiong ${ }^{*}$ and Jinghong Zhao* \\ Department of Nephrology, The Key Laboratory for the Prevention and Treatment of Chronic Kidney Disease of Chongqing, \\ Chongqing Clinical Research Center of Kidney and Urology Diseases, Xinqiao Hospital, Army Medical University (Third \\ Military Medical University), Chongaing, China
}

Aims: To investigate the potential role of renal arterial resistance index $(\mathrm{RI})$ in the differential diagnosis between diabetic kidney disease (DKD) and non-diabetic kidney disease (NDKD) and establish a better-quantified differential diagnostic model.

Materials and Methods: We consecutively reviewed 469 type 2 diabetes patients who underwent renal biopsy in our center. According to the renal biopsy results, eligible patients were classified into the DKD group and the NDKD group. The diagnostic significance of Rl was evaluated by receiver operating characteristic $(\mathrm{ROC})$ curve analysis. Logistic regression analysis was used to search for independent risk factors associated with DKD. Then a novel diagnostic model was established using multivariate logistic regression analysis.

Results: A total of 332 DKD and 137 NDKD patients were enrolled for analysis. RI was significantly higher in the DKD group compared with those in the NDKD group (0.70 vs. $0.63, p<0.001)$. The optimum cutoff value of $\mathrm{Rl}$ for predicting DKD was 0.66 with sensitivity (69.2\%) and specificity (80.9\%). Diabetic retinopathy, diabetes duration $\geq 60$ months, $\mathrm{HbA} 1 \mathrm{c} \geq 7.0(\%), \mathrm{Rl} \geq 0.66$, and body mass index showed statistical significance in the multivariate logistic regression analysis. Then, we constructed a new diagnostic model based on these results. And the validation tests indicated that the new model had good sensitivity (81.5\%) and specificity (78.6\%).

Conclusions: RI has a potential role in discriminating DKD from NDKD. The RI-based predicting model can be helpful for differential diagnosis of DKD and NDKD.

Keywords: diabetic kidney disease, non-diabetic kidney disease, resistance index, differential diagnosis, type 2 diabetes mellitus

\section{INTRODUCTION}

Diabetes mellitus (DM) is a global public health challenge affecting over 463 million adults, according to the report of the International Diabetes Federation in 2019 (1). In China, it is estimated that approximately $11.2 \%$ of the population has DM (129.8 million people) (2). Type 2 diabetes mellitus (T2DM) combined with renal impairment is correlated with increased cardiovascular 
mortality and all-cause mortality (3). Diabetic kidney disease (DKD) is now one of the most frequent and severe complications of diabetes and continues to be the principal cause of end-stage kidney disease (ESKD) worldwide $(4,5)$. However, non-diabetic kidney disease (NDKD) occurs in T2DM patients as well (6). The prevalence of NDKD in T2DM varied widely from $36.8 \%$ to $82.9 \%(7-12)$. The therapy and prognosis of NDKD are pretty different from DKD $(13,14)$. It is believed that the renal outcomes of patients with DKD are relatively worse compared with their counterparts with biopsy-proven NDKD because the pathological changes of DKD are deemed difficult to reverse (15, 16). Therefore, it is critical to distinguish between DKD and NDKD in diabetes with renal impairment in clinical practice.

Currently, the renal pathological diagnosis is the gold standard to discriminate DKD from NDKD. However, the kidney biopsy is an invasive procedure that is impracticable in patients with contraindications, such as pyknotic kidney, bleeding tendency, solitary kidney, uncontrolled hypertension, or severe anemia. Moreover, a renal biopsy could not be routinely performed in some primary hospitals. Thus, the diagnosis and appropriate treatment were usually based on clinical indicators, such as diabetes duration, hematuria, diabetic retinopathy (DR), glycated hemoglobin (HbAlc), and other indices (17-22). However, those markers are not entirely accurate. For instance, lack of DR contributes to the diagnosis of NDKD but does not rule out DKD (23). In recent years, some studies have used some new markers and diagnostic models for the clinical differentiation between DKD and NDKD, such as dysmorphic erythrocytes and urinary neutrophil gelatinase-associated lipocalin $(22,24)$. However, these markers or risk model is not perfect and is still not good enough to meet clinical requirements. Therefore, it is necessary to find a new precise and sensitive non-invasive marker for clinical differentiation of DKD from NDKD.

The Renal atrial resistance index (RI), measured by doppler ultrasound, is a low-cost and non-invasive tool in detecting kidney diseases; it has been extensively used to evaluate renal blood flow as a semi-quantitative parameter. Previous studies suggested that RI is correlated with severe interstitial fibrosis and the progression of chronic kidney disease (CKD) (25-27). In addition, a few studies have noticed that RI in patients with $\mathrm{DKD}$ is significantly higher when compared with non-diabetic controls, which might be helpful for the identification and prediction of DKD $(28,29)$. However, the potential role of RI in the clinical differentiation of DKD from NDKD and the optimal cutoff value remains unclear. Thus, the present study was conducted to investigate the potential role of RI in the differential diagnosis between DKD and NDKD and establish a better-quantified differential diagnostic model.

\section{MATERIALS AND METHODS}

\section{Study Subjects}

A total of 469 T2DM patients with renal impairment from the department of nephrology at Xinqiao Hospital, Army Medical University, from January 2014 to September 2020 were retrospectively analyzed (Figure 1). All patients had received echo-color-Doppler examination of renal vessels, systematic screening for diabetic retinopathy, and renal biopsy. The diagnosis of T2DM met the criteria proposed by American Diabetes Association in 2019 (30). Eligible patients were divided into the DKD group and the NDKD group based on the kidney biopsy results. The inclusion criteria were: T2DM patients with renal impairment and received kidney biopsy; serum creatinine < $442 \mu \mathrm{mol} / \mathrm{L}$. The exclusion criteria were: age above 75 years or below 18 years; lack of a fundus clinical information data and pathological data or clear medical history; severe complications, such as severe infection, heart failure, and hypertensive emergency; biopsy-proven DKD complicated by NDKD. All patients signed informed consent before kidney biopsy. The study was approved by the ethical committee of Xinqiao Hospital, and was in accordance with the principles of the Declaration of Helsinki.

\section{Renal Biopsy and Pathological Examination}

The kidney biopsies were performed by an experienced renal pathologist, and every kidney biopsy tissue was investigated by electron microscopy, light microscopy, and immunofluorescence. The kidney biopsy indications were in accordance with the KDOQI Guideline (31). The pathological diagnostic criteria for DKD was: diffuse mesangial proliferation, diffuse capillary glomerulosclerosis, presence of Kimmelstiel-Wilson nodular lesions, glomerular basement membrane thickening, hyaline exudative lesions (32). NDKD usually has some unique features based on the guidelines previously reported (12).

\section{Clinical and Laboratory Information}

The following data were collected at the time of kidney biopsy: age, sex, body mass index (BMI), systolic blood pressure, diastolic blood pressure, presence of hypertension and DR, medical history of DM, fasting blood glucose, HbA1c, hemoglobin, platelet, serum albumin, total cholesterol, triglyceride, serum creatinine, uric acid, blood urea nitrogen, 24-hour urine protein, hematuria, and RI value. Blood pressure was measured three times according to a standard protocol, and the average value was calculated. DR was confirmed by fundus photography. Ultrasonography was performed by a Philips IU22 Cart G Ultrasound System with C5-1(Made in United Kingdom). The estimated glomerular filtration rate (eGFR) was obtained using the chronic kidney disease epidemiology collaboration (CKD-EPI) equation (33).

\section{Statistical Analysis}

Continuous variables were shown as average \pm standard deviation or the median and interquartile depending on the data distribution, whereas enumeration data were described as percentages (\%). The ttest was applied for normally distributed data, and the MannWhitney $\mathrm{U}$ test was applied for non-normally distributed data. The chi-squared test was applied for enumeration data. Receiver operating characteristic (ROC) curve analysis was used to explore the optimal cutoff point of the RI to predict DKD. Univariate and multivariate logistic regression analyses (stepwise forward) were performed to search the independent risk factors relating to the 


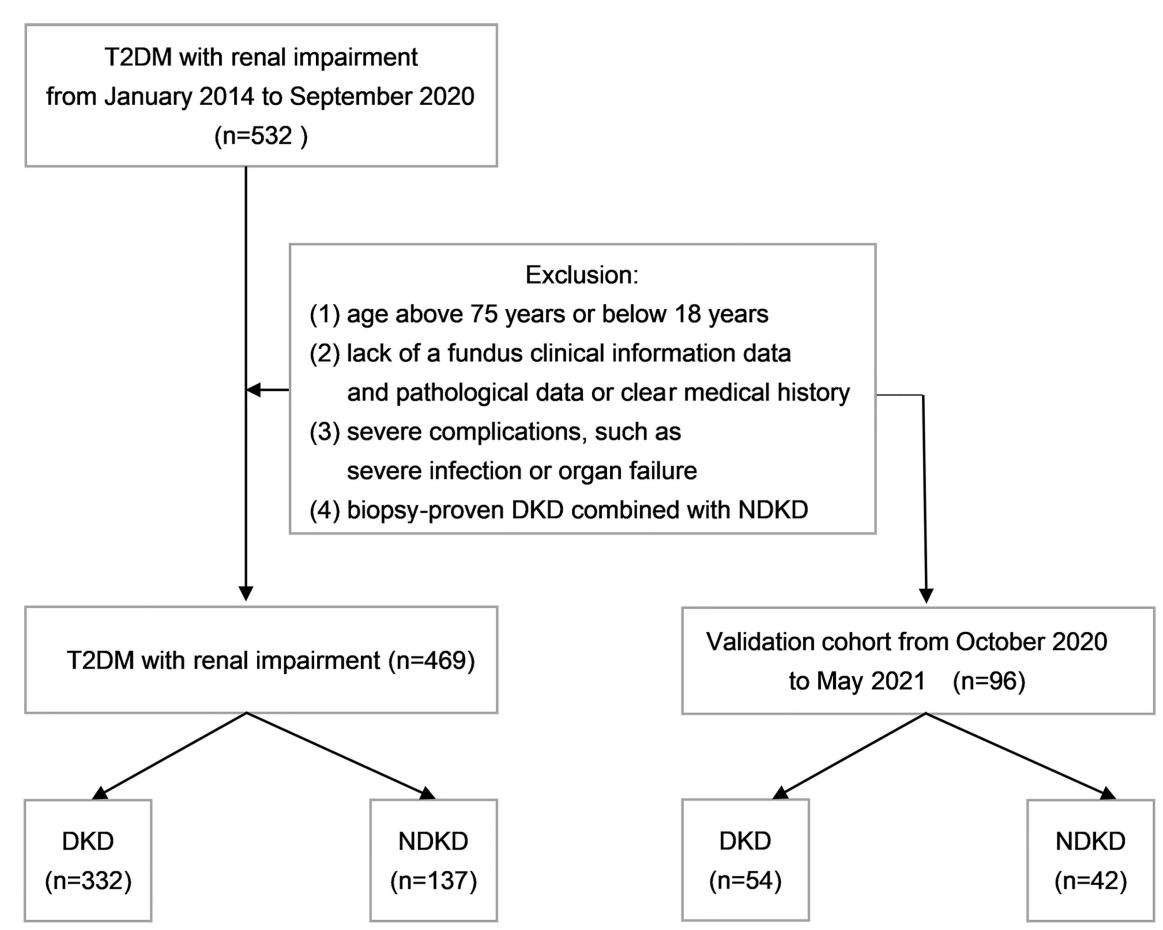

FIGURE 1 | Flowchart showing the procedure for the selection of study participants. T2DM, type 2 diabetes mellitus; DKD, diabetic kidney disease; NDKD, nondiabetic kidney disease.

DKD diagnosis, with results shown as the odds ratio (OR) and 95\% confidence interval (CI). The final significant risk factors were included in two differential diagnostic models (with or without $\mathrm{RI})$. The equation is as follows: $P D K D=\exp (\alpha+\beta 1 \times 1+\beta 2 \times 2+\beta 3 \times 3+$ $\ldots+\beta n \times n) /[1+\exp (\alpha+\beta 1 \times 1+\beta 2 \times 2+\beta 3 \times 3+\ldots+\beta n \times n)]$. PDKD is the probability of DKD diagnosis, $\alpha$ is a constant, $\beta$ is the estimator, and $\mathrm{x}$ is the clinical predictor. If $\mathrm{PDKD} \geq 0.5$, the patient should be considered as $\mathrm{DKD}$, while the diagnosis should be NDKD if $\mathrm{PDKD}<0.5$. The Delong test and the calculation of the net reclassification improvement and the integrated discrimination improvement were performed by $\mathrm{R}$ language to analyze two models. Then, a better model was selected. Finally, a back-substitution and a validation test (by a validation cohort of 96 cases) were conducted to evaluate the new model. Correlations between RI and clinical indices were analyzed by the Pearson test. Statistical analyses were performed by SPSS (IBM SPSS Statistics 23.0) and R language. $\mathrm{P}<0.05$ was considered statistically significant. The number of patients required for the validation cohort is computed using software PASS 15.0.5.

\section{RESULTS}

\section{The Clinical Characteristics and Renal Pathological Features of the Included Patients}

A total of 469 patients were divided into two groups based on kidney biopsy results, with 332 patients in the DKD group and 137 patients in the NDKD group. The general clinical information of the two groups was shown in Table 1. Compared with the NDKD group, patients in the $\mathrm{DKD}$ group had longer diabetes duration, higher incidence of DR, and higher levels of systolic blood pressure, fasting blood glucose, $\mathrm{HbAlc}$, serum creatine, blood urea nitrogen and RI value, while lower levels of BMI, hemoglobin, triglyceride and eGFR. But no significant difference was noticed between the two groups regarding age, gender, diastolic blood pressure, platelets, serum albumin, total cholesterol, uric acid, urinary protein, or presence of hematuria, nephrotic syndrome, hypertension, cardiovascular disease. Moreover, renal pathological findings showed that membranous nephropathy (38 cases, 27.74\%) and IgA nephropathy (38 cases, $27.74 \%$ ) were the most common pathological type among the $137 \mathrm{NDKD}$ patients, followed by mesangial proliferative glomerulonephritis (19 cases, 13.87\%), hypertensive nephrosclerosis $(16,11.68 \%)$, and other types (Supplementary Table 1). The main pathological manifestations of the DKD group were advanced lesions, with $71.08 \%$ of the total were classified as class III (Supplementary Table 2).

\section{Differential Diagnosis Performance of RI in Diabetic Patients With Renal Impairment}

To explore the clinical value of RI in DKD and NDKD, we compared the RI value in the two groups. Results showed patients in the DKD group had a significantly higher RI value compared with those in the NDKD group ( 0.70 vs. $0.63, p<0.001$, Table 1). Then, we performed a ROC curve to determine the cutoff point of $\mathrm{RI}$ for predicting DKD. The area under the curve (AUC) of RI was 0.785 . The best cutoff point of RI was 0.66 , with $69.2 \%$ sensitivity 
TABLE 1 | The general clinical characteristics of the included patients.

\begin{tabular}{|c|c|c|c|c|}
\hline Parameter & $\begin{array}{l}\text { All cases } \\
(n=469)\end{array}$ & $\begin{array}{c}\text { NDKD } \\
(n=137)\end{array}$ & $\begin{array}{c}\text { DKD } \\
(n=332)\end{array}$ & $P$ value \\
\hline Age, (years) & $51.53 \pm 10.26$ & $52.04 \pm 11.05$ & $51.32 \pm 9.93$ & 0.489 \\
\hline Gender, (male, \%) & $299(63.75)$ & $82(59.85)$ & 217 (65.36) & 0.259 \\
\hline BMl, $\left(\mathrm{kg} / \mathrm{m}^{2}\right)$ & $25.24 \pm 3.48$ & $26.51 \pm 4.01$ & $24.80 \pm 3.42$ & $<0.001$ \\
\hline $\mathrm{SBP},(\mathrm{mm} \mathrm{Hg})$ & $142.73 \pm 22.62$ & $136.62 \pm 21.42$ & $145.26 \pm 22.69$ & $<0.001$ \\
\hline $\mathrm{DBP},(\mathrm{mm} \mathrm{Hg})$ & $84.43 \pm 12.16$ & $84.32 \pm 12.58$ & $84.47 \pm 12.00$ & 0.905 \\
\hline Duration of diabetes, (months) & $76.03 \pm 62.41$ & $28.10 \pm 30.59$ & $94.66 \pm 61.71$ & $<0.001$ \\
\hline Duration of diabetes $\geq 60$ months (\%) & $240(51.17)$ & $23(16.79)$ & $217(65.35)$ & $<0.001$ \\
\hline $\mathrm{HbA} 1 \mathrm{c}$ & $7.82 \pm 2.14$ & $7.32 \pm 1.90$ & $8.01 \pm 2.19$ & 0.004 \\
\hline $\mathrm{HbA} 1 \mathrm{c} \geq 7,(\%)$ & $216(46.06)$ & 45 (32.85) & $171(51.51)$ & $<0.001$ \\
\hline FBG, (mmol/L) & $7.34 \pm 3.33$ & $6.76 \pm 2.92$ & $7.58 \pm 3.46$ & 0.015 \\
\hline Hemoglobin, (g/L) & $117.51 \pm 24.93$ & $127.02 \pm 22.02$ & $113.59 \pm 25.03$ & $<0.001$ \\
\hline PLT, (10^9/L) & $205.76 \pm 79.36$ & $206.44 \pm 80.91$ & $205.48 \pm 78.84$ & 0.906 \\
\hline Serum albumin, (g/L) & $35.05 \pm 9.43$ & $34.20 \pm 10.81$ & $35.40 \pm 8.79$ & 0.215 \\
\hline $\mathrm{TC},(\mathrm{mmol} / \mathrm{L})$ & $5.47 \pm 1.98$ & $5.65 \pm 2.45$ & $5.40 \pm 1.76$ & 0.217 \\
\hline TG, (mmol/L) & $2.21 \pm 1.86$ & $2.57 \pm 2.19$ & $2.07 \pm 1.69$ & 0.008 \\
\hline Uric acid, $(\mu \mathrm{mol} / \mathrm{L})$ & $378.59 \pm 96.49$ & $372.42 \pm 108.05$ & $381.04 \pm 91.57$ & 0.388 \\
\hline $\mathrm{Scr},(\mu \mathrm{mol} / \mathrm{L})$ & $127.32 \pm 76.47$ & $102.41 \pm 60.89$ & $137.52 \pm 79.87$ & $<0.001$ \\
\hline BUN, (mmol/L) & $7.86 \pm 3.67$ & $6.89 \pm 3.39$ & $8.26 \pm 3.71$ & $<0.001$ \\
\hline eGFR, $\left(\mathrm{ml} / \mathrm{min} / 1.73 \mathrm{~m}^{2}\right)$ & $68.08 \pm 35.44$ & $82.67 \pm 34.37$ & $61.32 \pm 34.23$ & $<0.001$ \\
\hline Urinary protein, (g/24 h) & $3.41 \pm 3.71$ & $3.49 \pm 4.39$ & $3.38 \pm 3.41$ & 0.778 \\
\hline Hematuria (\%) & 320 (68.23) & $96(70.07)$ & $224(67.47)$ & 0.372 \\
\hline $\mathrm{RI}$ & $0.68 \pm 0.07$ & $0.63 \pm 0.05$ & $0.70 \pm 0.07$ & $<0.001$ \\
\hline \multicolumn{5}{|l|}{ Clinical comorbidities } \\
\hline Nephrotic syndrome (\%) & 109 (23.24) & 32 (23.36) & 77 (23.19) & 0.969 \\
\hline Hypertension (\%) & 325 (69.29) & 90 (65.69) & 235 (70.78) & 0.282 \\
\hline Cardiovascular disease (\%) & 180 (38.38) & $52(37.96)$ & $128(38.55)$ & 0.799 \\
\hline $\mathrm{DR}(\%)$ & $227(48.40)$ & $12(8.76)$ & $215(64.76)$ & $<0.001$ \\
\hline
\end{tabular}

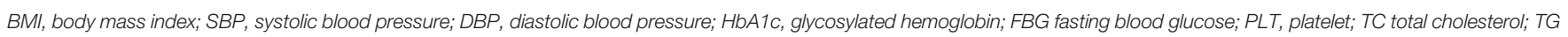

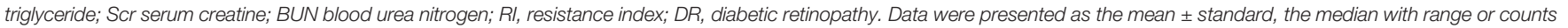
and percentages. A two-tailed $P<0.05$ was considered statistically significant.

and $80.9 \%$ specificity, as calculated by obtaining the best Youden index (Figure 2). Then, we assigned a value of 1 to $\mathrm{RI} \geq 0.66$ and converted it into a binary variable. Considering the value of $\mathrm{RI}$ in the differential diagnosis of two groups, the correlation analysis between $\mathrm{RI}$ and other clinical information was performed. There were 256 (54.6\%) patients with $\mathrm{RI} \geq 0.66$ and 213 (45.4\%) patients with $\mathrm{RI}<$ 0.66 , respectively. Compared with patients with $\mathrm{RI}<0.66$, patients with $\mathrm{RI} \geq 0.66$ had higher age, systolic blood pressure, duration of diabetes, serum creatinine, blood urea nitrogen, the proportion of hypertension, diabetic retinopathy, and nephrotic syndrome, while a lower level of BMI, serum albumin, hemoglobin, TG, and eGFR (Table 2). Further analyses was performed by linear regression, RI levels were positively correlated with age $(\mathrm{r}=0.245 ; p<0.001)$, duration of diabetes $(\mathrm{r}=0.341 ; p<0.001)$, systolic blood pressure $(\mathrm{r}=0.274 ; p<0.001)$; serum creatinine $(\mathrm{r}=0.335 ; p<0.001)$; blood urea nitrogen $(\mathrm{r}=0.037 ; p<0.001)$ and total urinary protein, $\mathrm{g} / 24 \mathrm{~h}$ $(\mathrm{r}=0.141 ; p=0.003)$, whereas it was inversely correlated with BMI $(\mathrm{r}=$ $-0.143 ; p=0.002)$, hemoglobin $(\mathrm{r}=-0.424 ; p<0.001)$, triglyceride $(\mathrm{r}=$ $-0.145 ; p=0.002)$, and serum albumin $(\mathrm{r}=-0.163 ; p<0.001)$ in diabetic patients with renal impairment (Supplementary Table 3).

\section{Screening for DKD Diagnosis-Related Factors}

Previous studies found that the duration of diabetes $\leq 60$ months was an independent risk factor for NDKD (11). Many researches indicated that intensive blood glucose control (HbA1c 6.5-7.0\%) could reduce the risk of DKD. HbAlc $<7.0$ was considered as a common indicator of clinical blood glucose control (34). Therefore, we assigned a value of 1 to the duration of diabetes $\geq$ 60 months and $\mathrm{HbAlc} \geq 7 \%$ respectively. Then those indicators were converted into binary variables. Univariate regression analysis indicated that duration of diabetes $\geq 60$ months, BMI, systolic blood pressure, DR, fasting blood glucose, HbA1c $\geq 7 \%$, hemoglobin, triglyceride, serum creatinine, blood urea nitrogen, and $\mathrm{RI} \geq 0.66$ were related to the diagnosis of DKD. After adjusting for the factors mentioned above using multivariate logistic regression analysis, $\mathrm{RI} \geq 0.66$ was still an independent risk factor for the DKD diagnosis, as well as the duration of diabetes $\geq 60$ months, BMI, DR, and HbAlc $\geq 7 \%$ (Table 3).

\section{Establishment and Validation of the New Differential Diagnostic Model}

Then, we used two multivariate logistic regression analyses to establish two differential diagnostic models (with or without RI value) to explore the RI value for the clinical differentiation between DKD and NDKD (Table 4). The traditional model was built by four independent risk factors other than RI, and the RIbased model was built by five independent risk factors, including $\mathrm{RI} \geq 0.66$. The detailed equation of the two models is shown in Supplementary Table 4. The area under ROC curve of the traditional model was 0.889. After adding RI, the area under the ROC curve of the RI-based model increased to 0.912 


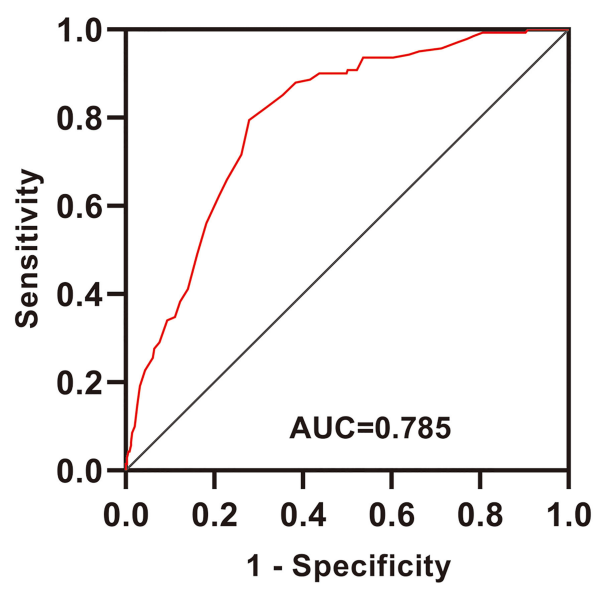

\begin{tabular}{ccccccc}
\hline & Cutoff & Sensitivity & Specificity & AUC $(95 \% \mathrm{Cl})$ & Youden index & P value \\
\hline RI & $\mathbf{0 . 6 6 0}$ & $69.2 \%$ & $80.9 \%$ & $0.785(0.745,0.829)$ & 0.501 & $P<0.001$ \\
\hline
\end{tabular}

FIGURE 2 | Differential diagnosis performance of RI in type 2 diabetic patients with renal impairment evaluated by ROC curve. RI, resistance index; AUC, the area under ROC curve; $\mathrm{Cl}$, confidence interval; $\mathrm{ROC}$, receiver operating characteristic.

(Figure 3A). The statistical significance of two ROC AUC by DeLong's test $(Z=2.5964, \mathrm{P}$ value $=0.00942)$, the net reclassification improvement $(\mathrm{NRI}=0.1837, \mathrm{P}$ value $=$ 0.00278 ), and the integrated discrimination improvement (IDI = $0.0572, \mathrm{P}$ value $=0.00013)$ implied that the RI-based model has improved the efficiency of differential diagnosis. The sample size of the validation cohort was computed by software PASS based on the AUC of the traditional model (AUC=0.912). The results showed that a random sample of 51 subjects from the positive population and 40 from the negative population produced a twosided $95.0 \%$ confidence interval with a width of 0.120 . Then, we recruited another 96 patients (from October 2020 to May 2021)

TABLE 2 | Comparison of clinical findings in T2DM with renal impairment according to renal resistance index.

\begin{tabular}{|c|c|c|c|}
\hline Parameters & $R I<0.66(n=213)$ & $R I \geq 0.66(n=256)$ & $P$ value \\
\hline Age, (years) & $49.20 \pm 10.47$ & $53.37 \pm 9.64$ & $<0.001$ \\
\hline Gender, (male, \%) & 132(61.97) & $166(64.84)$ & 0.526 \\
\hline BMl, $\left(\mathrm{kg} / \mathrm{m}^{2}\right)$ & $25.67 \pm 3.64$ & $24.87 \pm 3.31$ & 0.013 \\
\hline $\mathrm{SBP},(\mathrm{mm} \mathrm{Hg})$ & $136.73 \pm 20.47$ & $147.62 \pm 23.17$ & $<0.001$ \\
\hline $\mathrm{DBP},(\mathrm{mm} \mathrm{Hg})$ & $84.92 \pm 11.53$ & $83.96 \pm 12.69$ & 0.398 \\
\hline Duration of diabetes, (months) & $51.46 \pm 55.12$ & $95.69 \pm 61.01$ & $<0.001$ \\
\hline Duration of diabetes $\geq 60$, months (\%) & $70(32.86)$ & 169(66.01) & $<0.001$ \\
\hline $\mathrm{HbA1c},(\%)$ & $7.70 \pm 1.88$ & $7.94 \pm 2.33$ & 0.284 \\
\hline $\mathrm{HbA} 1 \mathrm{c} \geq 7,(\%)$ & $97(45.54)$ & $118(46.09)$ & 0.832 \\
\hline FBG, (mmol/L) & $7.17 \pm 3.08$ & $7.48 \pm 3.54$ & 0.313 \\
\hline Hemoglobin, (g/L) & $126.00 \pm 23.53$ & $110.60 \pm 23.95$ & $<0.001$ \\
\hline PLT, (10^9/L) & $211.45 \pm 82.92$ & $200.98 \pm 76.00$ & 0.157 \\
\hline Serum albumin, (g/L) & $36.13 \pm 10.45$ & $34.26 \pm 8.37$ & 0.034 \\
\hline $\mathrm{TG},(\mathrm{mmol} / \mathrm{L})$ & $2.44 \pm 1.97$ & $2.03 \pm 1.75$ & 0.018 \\
\hline $\mathrm{TC},(\mathrm{mmol} / \mathrm{L})$ & $5.47 \pm 2.24$ & $5.48 \pm 1.75$ & $0.920^{\circ}$ \\
\hline $\mathrm{Scr},(\mu \mathrm{mol} / \mathrm{L})$ & $106.47 \pm 60.54$ & $144.76 \pm 83.96$ & $<0.001$ \\
\hline eGFR, $\left(\mathrm{ml} / \mathrm{min} / 1.73 \mathrm{~m}^{2}\right)$ & $79.03 \pm 35.72$ & $59.22 \pm 32.71$ & $<0.001$ \\
\hline BUN, (mmol/L) & $7.01 \pm 3.21$ & $8.56 \pm 3.90$ & $<0.001$ \\
\hline Uric acid, $(\mu \mathrm{mol} / L)$ & $375.98 \pm 103.04$ & $381.33 \pm 91.05$ & 0.555 \\
\hline Urinary protein, (g/24 h) & $3.03 \pm 3.96$ & $3.73 \pm 3.47$ & 0.054 \\
\hline Hematuria (\%) & $134(62.91)$ & $184(71.88)$ & 0.056 \\
\hline Cardiovascular disease (\%) & 78 (36.61) & $101(39.45)$ & 0.479 \\
\hline Hypertension (\%) & $121(56.81)$ & $191(74.61)$ & 0.003 \\
\hline $\mathrm{DR}(\%)$ & 63 (29.58) & $163(63.67)$ & $<0.001$ \\
\hline Nephrotic syndrome (\%) & $40(18.78)$ & 69 (26.95) & 0.037 \\
\hline
\end{tabular}


TABLE 3 | Predictors of DKD in T2DM with renal impairment.

\begin{tabular}{|c|c|c|c|c|}
\hline \multirow[t]{2}{*}{ Parameters } & \multicolumn{2}{|c|}{ Univariate } & \multicolumn{2}{|c|}{ Multivariate } \\
\hline & OR $(95 \% \mathrm{Cl})$ & $P$ value & OR $(95 \% \mathrm{Cl})$ & $P$ value \\
\hline Duration of diabetes $\geq 60$ months (yes/no) & $9.34(5.61,15.56)$ & 0.000 & $5.29(2.63,10.59)$ & $<0.001$ \\
\hline $\mathrm{BMI},\left(\mathrm{kg} / \mathrm{m}^{2}\right)$ & $0.89(0.84,0.94)$ & 0.000 & $0.90(0.82,1.00)$ & 0.045 \\
\hline $\mathrm{SBP},(\mathrm{mmHg})$ & $1.02(1.01,1.03)$ & 0.001 & & \\
\hline DR (yes/no) & $19.14(10.16,36.07)$ & 0.000 & $14.80(6.40,34.26)$ & $<0.001$ \\
\hline $\mathrm{HbA1c} \geq 7 \%$ (yes/no) & $2.20(1.40,3.45)$ & 0.001 & $2.19(1.14,4.21)$ & 0.019 \\
\hline $\mathrm{FBG},(\mathrm{mmol} / \mathrm{L})$ & $1.09(1.02,1.17)$ & 0.016 & & \\
\hline Hemoglobin, (g/L) & $0.98(0.97,0.99)$ & 0.000 & & \\
\hline $\mathrm{TG},(\mathrm{mmol} / \mathrm{L})$ & $0.87(0.78,0.97)$ & 0.014 & & \\
\hline $\mathrm{Scr},(\mu \mathrm{mol} / \mathrm{L}$ & $1.01(1.01,1.01)$ & 0.000 & & \\
\hline BUN, (mmol/L) & $1.13(1.06,1.21)$ & 0.000 & & \\
\hline $\mathrm{RI} \geq 0.66$ (yes/no) & $9.50(5.84,15.46)$ & 0.000 & $5.19(2.59,10.38)$ & $<0.001$ \\
\hline
\end{tabular}

BMI, body mass index; SBP, systolic blood pressure; DR, Diabetic retinopathy; HbA1c, glycosylated hemoglobin; FBG, fasting blood glucose; TG, triglyceride; Scr, serum creatine; BUN, blood urea nitrogen; $\mathrm{Rl}$, resistance index; $\mathrm{Cl}$, confidence interval.

that met the same inclusion criteria to validate the RI-based model's efficacy further. The Validation cohort test demonstrated that the RI-based model had a sensitivity of $81.5 \%$ and a specificity of $78.6 \%$ (Table 5). The ROC curve (AUC=0.857) based on the validation cohort indicated that the RI-based model had good diagnostic efficiency (Figure 3B).

\section{DISCUSSION}

For T2DM patients with renal impairment, it is of great significance to differentiate between DKD and NDKD to guide clinical diagnosis and treatment. Kidney biopsy is the ultimate method of differential diagnosis, but its clinical application is restricted by many contraindications $(35,36)$. Therefore, it is necessary to find a new precise and sensitive non-invasive indicator for clinical differentiation of DKD from NDKD. The present study found that the RI value, which reflects vascular disease (37), was remarkably higher in the DKD group in contrast to the NDKD group. Furthermore, the RI-based differential model has good sensitivity and specificity.

Doppler ultrasound as a non-invasive, low-cost method has been extensively used in the detection of reno-vascular diseases. RI is calculated by the ratio of the difference between peak systolic velocity (PSV) and end-diastolic velocity (EDV) divided by peak systolic velocity (PSV), obtained by the Doppler spectrum analysis from segmental or interlobar arteries. PSV mainly reflects the degree of renal vascular filling and blood supply, while the EDV reflects renal blood perfusion, and RI mainly reflects vascular bed resistance (38). There is no uniform standard for RI average value. The normal mean renal RI value reported in previous literature are listed in Supplementary Table 5. Generally, a value of 0.60 is considered a normal value for renal RI $(39,40)$. Current studies have found that RI can effectively assess the renal blood perfusion status, whether for renal damage caused by hypertension and diabetes (41-43) or for risk prediction and disease assessment of early acute renal injury induced by various diseases (44-47). In patients with CKD, RI $\geq$ 0.70 is an independent risk factor for the progression of renal failure $(25,48)$. And $R I \geq 0.80$ was associated with a lower survival rate (49). Moreover, studies have also confirmed that the RI is higher in the newly diagnosed and untreated DKD patients than healthy controls $(28,29,50)$, even before the onset of microalbuminuria, supporting the dynamic evaluation of renal RI as an early detector of renal vascular alterations in the presence of T2DM (51). A previous study has also demonstrated a higher value of RI in DKD compared with diabetic patients without kidney disease (52). Different from previous studies, our study focused on comparing the RI level between the DKD group and the NDKD group. We found a significantly higher RI value in the DKD group. RI $\geq 0.66$ was proved to be an independent risk factor for the diagnoses of DKD in T2DM patients with renal impairment, which could improve the efficiency of differential diagnosis in identifying cases with a higher clinical suspicion for DKD. It is worth noting that the $\mathrm{RI}$ value is affected by age, race, region and many other factors so

TABLE 4 | Development of two differential diagnostic mode.

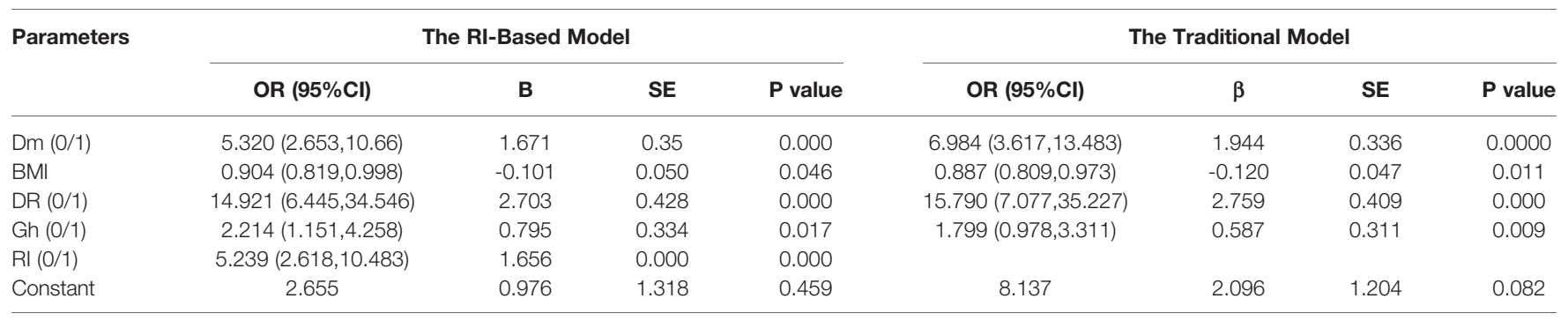

OR, odds ratio; SE, standard error; Dm, diabetes duration $\geq 60$ months (1 yes, O no); BMI, body mass index; DR, diabetic retinopathy (1 yes, 0 no); Gh, HbA1c $\geq 7.0 \%$ (1 yes, 0 no); RI, Resistance index $\geq 0.66$ ( 1 yes, 0 no). 
A

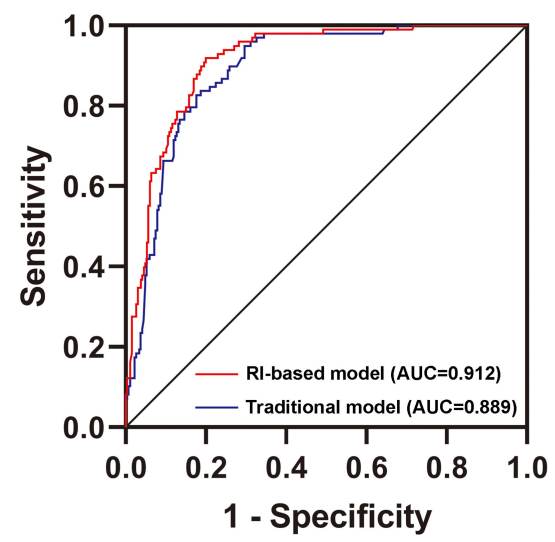

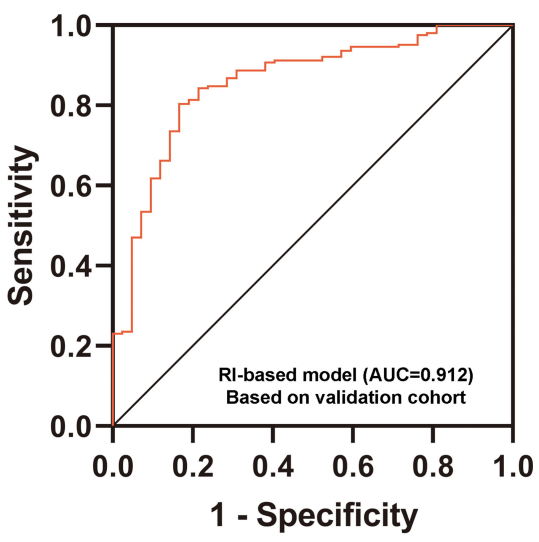

FIGURE 3 | Validation of the new differential diagnostic model. (A) Comparison of the area under the curve (AUC) of the RI-based model and the traditional model. DeLong's test was applied by R language to compare the AUC of two models (with or without RI). Z $=2.5964, P$ value $=0.00942$. (B) Receiver operating characteristics $(\mathrm{ROC})$ curve for the discriminative effect of the RI-based model in the Validation cohort. AUC, the area under the curve $=0.857$.

that the best cutoff value may be different in different studies. The cutoff value of RI in this study was derived from a small group of people in this specific region. A more appropriate cutoff value of RI needs further clinical research with a larger sample size in the future.

Glomerular hemodynamic changes (including hyperfiltration and hypoperfusion) are key pathological processes in the development of DKD, which could explain why RI value as an indicator of renal hemodynamics has a potential value in the differential diagnosis of DKD (53). The cause of glomerular hyperfiltration is currently believed to be caused by an increase in the trans-glomerular pressure gradient. The high glucose leads to the glycation of the basement of small blood vessels, particularly the efferent arteriole, making it thicken and stiffer and increasing the pressure within the glomerulus. Simultaneously, the afferent arteriole dilates, letting more blood flow into the glomeruli and increasing the pressure even further (54). With the deterioration of DKD, renal perfusion pressure and glomerular filtration rate continue to rise, resulting in glomerular capillary wall thickening, permeability enhancement, vascular lumen stenosis, glomerular capillary pressure increases. Those changes eventually lead to increased blood flow forward resistance and other renal artery hemodynamic disorders. Ultrasound showed that PSV and EDV decreased, especially EDV decreased and RI increased (53). Then, glomerular capillary basement membrane (GBM) thickening and mesangial matrix increased gradually, leading to glomerulosclerosis (55). Patients with NDKD often have less vascular involvement, such as membranous nephropathy mainly characterized by podocyte changes, and IgA nephropathy is primarily characterized by mesangial proliferation $(56,57)$. Although both DKD and NDKD patients suffered from renal hemodynamics changes, more significant vascular lesions and a higher RI value were detected in DKD patients.

Our research also found that DR and diabetic duration are powerful predictors of $\mathrm{DKD}$, which is consistent with previous studies $(11,18)$. Generally, the diabetes duration in DKD patients is longer than that in NDKD patients. Diabetes history $\geq 5$ years is an independent risk factor for DKD (58). In the present study, the diabetes duration is defined as the time from the diagnosis of diabetes to the time of renal biopsy. The diagnosis is usually delayed in patients with T2DM. It is hard to clarify their actual course of diabetes before diagnosis, so DKD cannot be ignored due to a short history of diabetes. Similarly, DR is related to DKD (59). CKD patients with $\mathrm{DR}$ are conducive to the diagnosis of $\mathrm{DKD}$, in which

TABLE 5 | Predictive value of the Back-substitution and Validation cohort test.

\begin{tabular}{|c|c|c|c|c|c|c|}
\hline & \multicolumn{3}{|c|}{ Back-Substitution Test } & \multicolumn{3}{|c|}{ Validation Cohort Test } \\
\hline & DKD & NDKD & Total & DKD & NDKD & Total \\
\hline Diagnosed as DKD & 300 & 45 & 345 & 44 & 9 & 53 \\
\hline Diagnosed as NDKD & 32 & 92 & 124 & 10 & 33 & 43 \\
\hline Total & 332 & 137 & 469 & 54 & 42 & 96 \\
\hline Sensitivity & & $90.4 \%$ & & & $81.5 \%$ & \\
\hline Specificity & & $67.2 \%$ & & & $78.6 \%$ & \\
\hline Positive predictive value & & $87.0 \%$ & & & $83.0 \%$ & \\
\hline Negative predictive value & & $74.2 \%$ & & & $76.7 \%$ & \\
\hline Total consistency & & $83.6 \%$ & & & $80.2 \%$ & \\
\hline
\end{tabular}

$D K D$, diabetic kidney disease; NDKD, non-diabetic kidney disease. 
proliferative retinopathy has higher specificity. DR often accompanies kidney damage in T1DM, but the incidence of DR in T2DM is only $40 \%-60 \%$. DR cannot completely distinguish DKD from NDKD because some diabetic patients without DR also have biopsy-proven DKD. Therefore, we choose to combine previous classic predictive indicators and RI that reflect vascular disease to construct a diagnostic model, significantly improving diagnostic efficiency. In addition, RI obtained by ultrasound examinations can be easily performed in most medical institutions, making our model more practical and economical in clinical practice. Therefore, we propose to incorporate the RI measure in the examination of DM combined with renal impairment. Interestingly, our study found for the first time that lower BMI was an independent risk factor for DKD. BMI was significantly lower in the DKD group than the NDKD group, which may be due to a longer duration of diabetes in the DKD group. However, compared with other independent risk factors, the OR value of BMI was only 0.9. Further studies are needed to power it as an independent risk factor for DKD.

There are several limitations in the present research. First of all, this research was a retrospective study performed in a single center with limited data. Secondly, all validation cohort patients came from the department of nephrology of Xinqiao Hospital, lacking external validation. Thirdly, we only measure RI value at the time of kidney biopsy, not continuous testing; certain errors are unavoidable.

In conclusion, the RI value might serve as a novel potential indicator in the differential diagnosis of DKD. The RI-based differential diagnostic model has improved the accuracy and could be commonly used in clinical practice.

\section{DATA AVAILABILITY STATEMENT}

The original contributions presented in the study are included in the article/Supplementary Material. Further inquiries can be directed to the corresponding authors.

\section{REFERENCES}

1. Zheng Y, Ley SH, Hu FB. Global Aetiology and Epidemiology of Type 2 Diabetes Mellitus and its Complications. Nat Rev Endocrinol (2018) 14(2):8898. doi: 10.1038/nrendo.2017.151

2. Li Y, Teng D, Shi X, Qin G, Qin Y, Quan H, et al. Prevalence of Diabetes Recorded in Mainland China Using 2018 Diagnostic Criteria From the American Diabetes Association: National Cross Sectional Study. BMJ (Clinical Res ed) (2020) 369:m997. doi: 10.1136/bmj.m997

3. Afkarian M, Sachs MC, Kestenbaum B, Hirsch IB, Tuttle KR, Himmelfarb J, et al. Kidney Disease and Increased Mortality Risk in Type 2 Diabetes. J Am Soc Nephrol (2013) 24(2):302-8. doi: 10.1681/asn.2012070718

4. Umanath K, Lewis JB. Update on Diabetic Nephropathy: Core Curriculum 2018. Am J Kidney Dis (2018) 71(6):884-95. doi: 10.1053/j.ajkd.2017.10.026

5. Ma RCW. Epidemiology of Diabetes and Diabetic Complications in China. Diabetologia (2018) 61(6):1249-60. doi: 10.1007/s00125-018-4557-7

6. Yan S-T, Liu J-Y, Tian H, Li C-L, Li J, Shao Y-H, et al. Clinical and Pathological Analysis of Renal Damage in Elderly Patients With Type 2 Diabetes Mellitus. Clin Exp Med (2016) 16(3):437-42. doi: 10.1007/s10238015-0362-5

7. Pham TT, Sim JJ, Kujubu DA, Liu IL, Kumar VA. Prevalence of Nondiabetic Renal Disease in Diabetic Patients. Am J Nephrol (2007) 27(3):322-8. doi: $10.1159 / 000102598$

\section{ETHICS STATEMENT}

The studies involving human participants were reviewed and approved by The ethical committee of Xinqiao Hospital. The patients/participants provided their written informed consent to participate in this study.

\section{AUTHOR CONTRIBUTIONS}

HL and YS carried out the studies, participated in data collection, statistical analysis, and the writing of the manuscript. ZY and YH contributed to the data analysis. TH, TX, and YL contributed to the data collection. JZ and JX reviewed and edited the manuscript. All authors contributed to the article and approved the submitted version.

\section{FUNDING}

This study was supported by research grants from Personal Training Program for Clinical Medicine Research of Army Medical University (No. 2018XLC1007), Frontier specific projects of Xinqiao Hospital (No. 2018YQYLY004), Scientific Renovation Project of Army Medical University (No. 2019XLC3038), and Clinical Medical Research Talents Project of Army Military Medical University (No. 2019XLC3028).

\section{SUPPLEMENTARY MATERIAL}

The Supplementary Material for this article can be found online at: https://www.frontiersin.org/articles/10.3389/fendo.2021.731187/ full\#supplementary-material

8. Mou S, Wang Q, Liu J, Che X, Zhang M, Cao L, et al. Prevalence of NonDiabetic Renal Disease in Patients With Type 2 Diabetes. Diabetes Res Clin Pract (2010) 87(3):354-9. doi: 10.1016/j.diabres.2009.11.012

9. Zhuo L, Ren W, Li W, Zou G, Lu J. Evaluation of Renal Biopsies in Type 2 Diabetic Patients With Kidney Disease: A Clinicopathological Study of 216 Cases. Int Urol Nephrol (2013) 45(1):173-9. doi: 10.1007/s11255-012-0164-6

10. Li L, Zhang X, Li Z, Zhang R, Guo R, Yin Q, et al. Renal Pathological Implications in Type 2 Diabetes Mellitus Patients With Renal Involvement. J Diabetes Complications (2017) 31(1):114-21. doi: 10.1016/j.jdiacomp.2016.10.024

11. Dong Z, Wang Y, Qiu Q, Zhang X, Zhang L, Wu J, et al. Clinical Predictors Differentiating Non-Diabetic Renal Diseases From Diabetic Nephropathy in a Large Population of Type 2 Diabetes Patients. Diabetes Res Clin Pract (2016) 121:112-8. doi: 10.1016/j.diabres.2016.09.005

12. Sharma SG, Bomback AS, Radhakrishnan J, Herlitz LC, Stokes MB, Markowitz GS, et al. The Modern Spectrum of Renal Biopsy Findings in Patients With Diabetes. Clin J Am Soc Nephrol (2013) 8(10):1718-24. doi: $10.2215 /$ cjn. 02510213

13. Anders HJ, Huber TB, Isermann B, Schiffer M. CKD in Diabetes: Diabetic Kidney Disease Versus Nondiabetic Kidney Disease. Nat Rev Nephrol (2018) 14(6):361-77. doi: 10.1038/s41581-018-0001-y

14. Chen Y, Lee K, Ni Z, He JC. Diabetic Kidney Disease: Challenges, Advances, and Opportunities. Kidney Dis (Basel Switzerland) (2020) 6(4):215-25. doi: 10.1159/000506634 
15. Bermejo S, González E, López-Revuelta K, Ibernon M, López D, MartínGómez A, et al. Risk Factors for non-Diabetic Renal Disease in Diabetic Patients. Clin Kidney J (2020) 13(3):380-8. doi: 10.1093/ckj/sfz177

16. Chang TI, Park JT, Kim JK, Kim SJ, Oh HJ, Yoo DE, et al. Renal Outcomes in Patients With Type 2 Diabetes With or Without Coexisting non-Diabetic Renal Disease. Diabetes Res Clin Pract (2011) 92(2):198-204. doi: 10.1016/ j.diabres.2011.01.017

17. Xu J, Hu XF, Huang W, Shen PY, Zhang W, Ren H, et al. [The Clinicopathological Characteristics of Diabetic Nephropathy and NonDiabetic Renal Diseases in Diabetic Patients]. Zhonghua Nei Ke Za Zhi (2017) 56(12):924-9. doi: 10.3760/cma.j.issn.0578-1426.2017.12.007

18. Zhou J, Chen X, Xie Y, Li J, Yamanaka N, Tong X. A Differential Diagnostic Model of Diabetic Nephropathy and Non-Diabetic Renal Diseases. Nephrol Dial Transplant (2007) 23(6):1940-5. doi: 10.1093/ndt/gfm897

19. Liu MY, Chen XM, Sun XF, Zhou JH, Zhang XG, Zhu HY, et al. Validation of a Differential Diagnostic Model of Diabetic Nephropathy and Non-Diabetic Renal Diseases and the Establishment of a New Diagnostic Model. J Diabetes (2014) 6(6):519-26. doi: 10.1111/1753-0407.12150

20. Liang S, Zhang XG, Cai GY, Zhu HY, Zhou JH, Wu J, et al. Identifying Parameters to Distinguish Non-Diabetic Renal Diseases From Diabetic Nephropathy in Patients With Type 2 Diabetes Mellitus: A Meta-Analysis. PloS One (2013) 8(5):e64184. doi: 10.1371/journal.pone.0064184

21. Mak S, Gwi E, Chan K, Wong P, Lo K, Lee K, et al. Clinical Predictors of NonDiabetic Renal Disease in Patients With non-Insulin Dependent Diabetes Mellitus. Nephrol Dialysis Transplant (1997) 12(12):2588-91. doi: 10.1093/ ndt/12.12.2588

22. Duan S, Chen J, Wu L, Nie G, Sun L, Zhang C, et al. Assessment of Urinary NGAL for Differential Diagnosis and Progression of Diabetic Kidney Disease. J Diabetes Complications (2020) 34(10):107665. doi: 10.1016/j.jdiacomp.2020.107665

23. Prakash J, Gupta T, Prakash S, Bhushan P, Usha, Sivasankar M, et al. NonDiabetic Renal Disease in Type 2 Diabetes Mellitus: Study of Renal - Retinal Relationship. Indian J Nephrol (2015) 25(4):222-8. doi: 10.4103/09714065.144420

24. Dong ZY, Wang YD, Qiu Q, Hou K, Zhang L, Wu J, et al. Dysmorphic Erythrocytes Are Superior to Hematuria for Indicating Non-Diabetic Renal Disease in Type 2 Diabetics. J Diabetes Investig (2016) 7(1):115-20. doi: $10.1111 /$ jdi.12371

25. Sugiura T, Wada A. Resistive Index Predicts Renal Prognosis in Chronic Kidney Disease: Results of a 4-Year Follow-Up. Clin Exp Nephrol (2011) 15 (1):114-20. doi: 10.1007/s10157-010-0371-3

26. Sugiura T, Wada A. Resistive Index Predicts Renal Prognosis in Chronic Kidney Disease. Nephrol Dialysis Transplant (2009) 24(9):2780-5. doi: 10.1093/ndt/gfp121

27. Bigé N, Lévy PP, Callard P, Faintuch JM, Chigot V, Jousselin V, et al. Renal Arterial Resistive Index Is Associated With Severe Histological Changes and Poor Renal Outcome During Chronic Kidney Disease. BMC Nephrol (2012) 13:139. doi: 10.1186/1471-2369-13-139

28. Ishimura E, Nishizawa Y, Kawagishi T, Okuno Y, Kogawa K, Fukumoto S, et al. Intrarenal Hemodynamic Abnormalities in Diabetic Nephropathy Measured by Duplex Doppler Sonography. Kidney Int (1997) 51(6):1920-7. doi: 10.1038/ki.1997.261

29. Masulli M, Mancini M, Liuzzi R, Daniele S, Mainenti PP, Vergara E, et al. Measurement of the Intrarenal Arterial Resistance Index for the Identification and Prediction of Diabetic Nephropathy. Nutr Metab Cardiovasc Dis (2009) 19(5):358-64. doi: 10.1016/j.numecd.2008.07.003

30. American Diabetes Association. Diabetes Advocacy: Standards of Medical Care in Diabetes-2019. Diabetes Care. (2019) 42(Suppl1):S182-s3. doi: $10.2337 / \mathrm{dc} 19-$ S016

31. National Kidney Foundation. KDOQI Clinical Practice Guideline for Diabetes and CKD: 2012 Update. Am J Kidney Dis (2012) 60(5):850-86. doi: 10.1053/ j.ajkd.2012.07.005

32. Tervaert TW, Mooyaart AL, Amann K, Cohen AH, Cook HT, Drachenberg CB, et al. Pathologic Classification of Diabetic Nephropathy. J Am Soc Nephrol (2010) 21(4):556-63. doi: 10.1681/asn.2010010010

33. Levey AS, Bosch JP, Lewis JB, Greene T, Rogers N, Roth D. A More Accurate Method to Estimate Glomerular Filtration Rate From Serum Creatinine: A New Prediction Equation. Modification of Diet in Renal Disease Study Group. Ann Intern Med (1999) 130(6):461-70. doi: 10.7326/0003-4819-130-6199903160-00002
34. Jiang W, Wang J, Shen X, Lu W, Wang Y, Li W, et al. Establishment and Validation of a Risk Prediction Model for Early Diabetic Kidney Disease Based on a Systematic Review and Meta-Analysis of 20 Cohorts. Diabetes Care (2020) 43(4):925-33. doi: 10.2337/dc19-1897

35. Kumar S, Lim E, Covic A, Verhamme P, Gale CP, Camm AJ, et al. Anticoagulation in Concomitant Chronic Kidney Disease and Atrial Fibrillation: JACC Review Topic of the Week. J Am Coll Cardiol (2019) 74 (17):2204-15. doi: 10.1016/j.jacc.2019.08.1031

36. Carey IM, Critchley JA, DeWilde S, Harris T, Hosking FJ, Cook DG. Risk of Infection in Type 1 and Type 2 Diabetes Compared With the General Population: A Matched Cohort Study. Diabetes Care (2018) 41(3):513-21. doi: $10.2337 / \mathrm{dc} 17-2131$

37. Di Nicolò P, Granata A. Renal Intraparenchymal Resistive Index: The Ultrasonographic Answer to Many Clinical Questions. J Nephrol (2019) 32 (4):527-38. doi: 10.1007/s40620-018-00567-x

38. Spatola L, Andrulli S. Doppler Ultrasound in Kidney Diseases: A Key Parameter in Clinical Long-Term Follow-Up. J Ultrasound (2016) 19 (4):243-50. doi: 10.1007/s40477-016-0201-x

39. Le Dorze M, Bouglé A, Deruddre S, Duranteau J. Renal Doppler Ultrasound: A New Tool to Assess Renal Perfusion in Critical Illness. Shock (2012) 37 (4):360-5. doi: 10.1097/SHK.0b013e3182467156

40. Keogan MT, Kliewer MA, Hertzberg BS, DeLong DM, Tupler RH, Carroll BA. Renal Resistive Indexes: Variability in Doppler US Measurement in a Healthy Population. Radiology (1996) 199(1):165-9. doi: 10.1148/radiology.199.1.8633141

41. Doi Y, Iwashima Y, Yoshihara F, Kamide K, Hayashi S, Kubota Y, et al. Response to Renal Resistive Index and Cardiovascular and Renal Outcomes in Essential Hypertension. Hypertension (2013) 61(2):e23. doi: 10.1161/ hypertensionaha.111.00664

42. Iwakura Y, Ito S, Morimoto R, Kudo M, Ono Y, Nezu M, et al. Renal Resistive Index Predicts Postoperative Blood Pressure Outcome in Primary Aldosteronism. Hypertension (2016) 67(3):654-60. doi: 10.1161/ hypertensionaha.115.05924

43. Ikee R, Kobayashi S, Hemmi N, Imakiire T, Kikuchi Y, Moriya H, et al. Correlation Between the Resistive Index by Doppler Ultrasound and Kidney Function and Histology. Am J Kidney Dis (2005) 46(4):603-9. doi: 10.1053/ j.ajkd.2005.06.006

44. Darmon M, Schortgen F, Vargas F, Liazydi A, Schlemmer B, Brun-Buisson C, et al. Diagnostic Accuracy of Doppler Renal Resistive Index for Reversibility of Acute Kidney Injury in Critically Ill Patients. Intensive Care Med (2011) 37 (1):68-76. doi: 10.1007/s00134-010-2050-y

45. Giustiniano E, Meco M, Morenghi E, Ruggieri N, Cosseta D, Cirri S, et al. May Renal Resistive Index be an Early Predictive Tool of Postoperative Complications in Major Surgery? Preliminary Results. BioMed Res Int (2014) 2014:917985. doi: 10.1155/2014/917985

46. Song J, Wu W, He Y, Lin S, Zhu D, Zhong M. Value of the Combination of Renal Resistance Index and Central Venous Pressure in the Early Prediction of Sepsis-Induced Acute Kidney Injury. J Crit Care (2018) 45:204-8. doi: 10.1016/j.jcrc.2018.03.016

47. Lerolle N, Guérot E, Faisy C, Bornstain C, Diehl JL, Fagon JY. Renal Failure in Septic Shock: Predictive Value of Doppler-Based Renal Arterial Resistive Index. Intensive Care Med (2006) 32(10):1553-9. doi: 10.1007/s00134-0060360-x

48. Parolini C, Noce A, Staffolani E, Giarrizzo GF, Costanzi S, Splendiani G. Renal Resistive Index and Long-Term Outcome in Chronic Nephropathies. Radiology (2009) 252(3):888-96. doi: 10.1148/radiol.2523080351

49. Radermacher J, Ellis S, Haller H. Renal Resistance Index and Progression of Renal Disease. Hypertension (2002) 39(2 Pt 2):699-703. doi: 10.1161/hy0202.103782

50. Brkljacic B, Mrzljak V, Drinkovic I, Soldo D, Sabljar-Matovinovic M, Hebrang A. Renal Vascular Resistance in Diabetic Nephropathy: Duplex Doppler US Evaluation. Radiology (1994) 192(2):549-54. doi: 10.1148/radiology.192. 2.8029430

51. Bruno RM, Daghini E, Landini L, Versari D, Salvati A, Santini E, et al. Dynamic Evaluation of Renal Resistive Index in Normoalbuminuric Patients With Newly Diagnosed Hypertension or Type 2 Diabetes. Diabetologia (2011) 54(9):2430-9. doi: 10.1007/s00125-011-2148-y

52. Platt JF, Rubin JM, Ellis JH. Diabetic Nephropathy: Evaluation With Renal Duplex Doppler US. Radiology (1994) 190(2):343-6. doi: 10.1148/ radiology.190.2.8284379 
53. Rossing K, Christensen PK, Hovind P, Tarnow L, Rossing P, Parving HH. Progression of Nephropathy in Type 2 Diabetic Patients. Kidney Int (2004) 66 (4):1596-605. doi: 10.1111/j.1523-1755.2004.00925.x

54. Tonneijck L, Muskiet MH, Smits MM, van Bommel EJ, Heerspink HJ, van Raalte DH, et al. Glomerular Hyperfiltration in Diabetes: Mechanisms, Clinical Significance, and Treatment. J Am Soc Nephrol (2017) 28(4):1023-39. doi: 10.1681/asn.2016060666

55. Wolf G, Butzmann U, Wenzel UO. The Renin-Angiotensin System and Progression of Renal Disease: From Hemodynamics to Cell Biology. Nephron Physiol (2003) 93(1):P3-13. doi: 10.1159/000066656

56. Cattran DC, Brenchley PE. Membranous Nephropathy: Integrating Basic Science Into Improved Clinical Management. Kidney Int (2017) 91(3):566-74. doi: 10.1016/j.kint.2016.09.048

57. Suzuki H, Kiryluk K, Novak J, Moldoveanu Z, Herr AB, Renfrow MB, et al. The Pathophysiology of IgA Nephropathy. J Am Soc Nephrol (2011) 22 (10):1795-803. doi: 10.1681/asn.2011050464

58. Yenigun EC, Dede F, Ozturk R, Turgut D, Koc E, Piskinpasa SV, et al. NonDiabetic Renal Disease in Diabetes Mellitus: Clinical Features and Renal Biopsy Findings. Hippokratia (2015) 19(2):148-52.

59. Fiorentino M, Bolignano D, Tesar V, Pisano A, Biesen WV, Tripepi G, et al. Renal Biopsy in Patients With Diabetes: A Pooled Meta-Analysis of 48
Studies. Nephrol Dial Transplant (2017) 32(1):97-110. doi: 10.1093/ndt/ gfw070

Conflict of Interest: The authors declare that the research was conducted in the absence of any commercial or financial relationships that could be construed as a potential conflict of interest.

Publisher's Note: All claims expressed in this article are solely those of the authors and do not necessarily represent those of their affiliated organizations, or those of the publisher, the editors and the reviewers. Any product that may be evaluated in this article, or claim that may be made by its manufacturer, is not guaranteed or endorsed by the publisher.

Copyright (c) 2022 Li, Shen, Yu, Huang, He, Xiao, Li, Xiong and Zhao. This is an open-access article distributed under the terms of the Creative Commons Attribution License (CC BY). The use, distribution or reproduction in other forums is permitted, provided the original author(s) and the copyright owner(s) are credited and that the original publication in this journal is cited, in accordance with accepted academic practice. No use, distribution or reproduction is permitted which does not comply with these terms. 